\title{
A strong generic ergodicity property of unitary and self-adjoint operators
}

\author{
A. S. KECHRIS and N. E. SOFRONIDIS \\ Department of Mathematics, Caltech, Pasadena, CA 91125, USA \\ (e-mail: kechris@caltech.edu) \\ (Received 11 June 1999 and accepted in revised form 14 August 2000)
}

\begin{abstract}
Consider the conjugacy action of the unitary group of an infinite-dimensional separable Hilbert space on the unitary operators. A strong generic ergodicity property of this action is established, by showing that any conjugacy invariants assigned in a definable way to unitary operators, and taking as values countable structures up to isomorphism, generically trivialize. Similar results are proved for conjugacy of self-adjoint operators and for measure equivalence. The proofs make use of the theory of turbulence for continuous actions of Polish groups, developed by Hjorth. These methods are also used to give a new solution to a problem of Mauldin in measure theory, by showing that any analytic set of pairwise orthogonal measures on the Cantor space is orthogonal to a product measure.
\end{abstract}

\section{Introduction}

Two of the main classes of operators on the infinite-dimensional separable complex Hilbert space $\mathbf{H}$, which are studied extensively in functional analysis and operator theory, are the unitary and self-adjoint operators. The unitary operators equipped with the strong (equivalently, the weak) operator topology form a Polish (i.e. completely metrizable separable) group $U(\mathbf{H})$ under multiplication, while the self-adjoint operators with norm at most 1 form a Polish space $S_{1}(\mathbf{H})$, when equipped with the strong topology.

The unitary group $U(\mathbf{H})$ acts continuously on both $U(\mathbf{H})$ and $S_{1}(\mathbf{H})$ by conjugation, and our main purpose in this paper is to study (unitary) conjugacy invariants of unitary and self-adjoint operators, i.e. functions $f: U(\mathbf{H}) \rightarrow X$ and $g: S_{1}(\mathbf{H}) \rightarrow Y$ with the property that $f\left(V U V^{-1}\right)=f(U)$ and $g\left(V S V^{-1}\right)=g(S)$, whenever $U, V$ are in $U(\mathbf{H})$ and $S$ is in $S_{1}(\mathbf{H})$. For example, any such $f$ and $g$, for which $f(U)$ and $g(S)$ depend only on the spectrum or the point spectrum of $U$ and $S$ respectively, constitute conjugacy invariants.

The spectral theorem for unitary operators provides a complete invariant under conjugacy, which consists of: 
(1) an equivalence class of probability Borel measures on the unit circle $\mathbf{T}$, under the measure equivalence relation defined by: $\mu \sim v$ iff $\mu$ and $v$ have the same null sets;

(2) a multiplicity function.

Similarly in the case of self-adjoint operators in $S_{1}(\mathbf{H})$ but with $[-1,1]$ replacing $\mathbf{T}$. If we restrict ourselves just to the set of multiplicity-free (i.e. with spectral multiplicity 1) unitary (respectively, self-adjoint) operators, which forms a dense $G_{\delta}$ set in $U(\mathbf{H})$ (respectively, $S_{1}(\mathbf{H})$ ) (see [1-3]), then this complete invariant is just a measure class on $\mathbf{T}$ (respectively, $[-1,1])$.

Our main purpose in this paper is to show that certain kinds of invariants are as far from complete as possible. For example, consider the following well-known instance of this phenomenon. Suppose that $f: U(\mathbf{H}) \rightarrow X$ is a nice, i.e. Baire measurable, function from $U(\mathbf{H})$ into some Polish space $X$. If $f$ is conjugacy invariant, then it is constant on a comeager set. This is a special case of the following standard topological ergodicity property (see, e.g., $[\mathbf{4}, 8.46])$ : if a group $G$ acts by homeomorphisms on a Baire space $Y$ and at least one orbit is dense, then for any Polish space $X$, every $G$-invariant $f: Y \rightarrow X$ is constant on a co-meager set. In our case, $G=U(\mathbf{H}), Y=U(\mathbf{H})$ and $U(\mathbf{H})$ acts by conjugation on itself. As shown in [1], the conjugacy class of every unitary operator whose spectrum is the entire unit circle is dense in $U(\mathbf{H})$. (A similar result also holds for $f: S_{1}(\mathbf{H}) \rightarrow X$ as above.)

In what follows, we denote by $S_{\infty}$ the infinite symmetric group, namely the Polish group of all permutations of the set $\mathbb{N}$ of natural numbers. For any Polish group $G$, by a Borel $G$-space we mean a Polish space $X$ together with a Borel action of $G$ on $X$. We denote by $E_{G}^{X}$ the corresponding orbit equivalence relation:

$$
x E_{G}^{X} y \Longleftrightarrow(\exists g \in G)(g \cdot x=y) .
$$

Our main result is the following very strong form of generic ergodicity for the action of $U(\mathbf{H})$ on $U(\mathbf{H}), S_{1}(\mathbf{H})$ by conjugation.

THEOREM 1. If $X$ is any Borel $S_{\infty}$-space and $f: U(\mathbf{H}) \rightarrow X$ is any Baire measurable function with the property that $U \approx V \Rightarrow f(U) E_{S_{\infty}}^{X} f(V)$, where $U \approx V$ means that the operators $U$ and $V$ are conjugate, then there exists a conjugacy invariant dense $G_{\delta}$ subset $A$ of $U(\mathbf{H})$ such that $f[A]$ is contained in a single $E_{S_{\infty}}^{X}$-class (i.e. a single $S_{\infty}$-orbit). Similarly for $S_{1}(\mathbf{H})$.

Remark. It should be pointed out here that every conjugacy class in $U(\mathbf{H}), S_{1}(\mathbf{H})$ is meager (see $[\mathbf{1}, \mathbf{2}])$.

Thus, any conjugacy invariants (computed in a Baire measurable way) which can be represented as orbits of an $S_{\infty}$-action must generically trivialize. Here are a couple of examples of such possible invariants.

(i) Countable subsets of a Polish space $S$. Let $f: U(\mathbf{H}) \rightarrow P_{\aleph_{0}}(S)\left(P_{\aleph_{0}}(S)\right.$ denoting the set of all countable subsets of $S$ ) be an arbitrary conjugacy invariant mapping, in the sense that $U \approx V \Rightarrow f(U)=f(V)$, whenever $U$ and $V$ are in $U(\mathbf{H})$. (An example of such a mapping would be the assignment of the point spectrum, i.e. the set of all its eigenvalues, to every operator in $U(\mathbf{H})$.) There are several standard ways to find a Borel 
$S_{\infty}$-space $X$ in which the orbits formed by the action of $S_{\infty}$ are in a canonical bijection $\pi: X / S_{\infty} \rightarrow P_{\aleph_{0}}(S)$ (see, e.g., [5]). It is natural then to consider $f$ nice if it has a Baire measurable lifting $f^{*}: U(\mathbf{H}) \rightarrow X$, in the sense that $\pi\left(f^{*}(U)\right)=f(U)$, whenever $U \in U(\mathbf{H})$. Then, for any $U, V$ in $U(\mathbf{H}), U \approx V \Rightarrow f^{*}(U) E_{S_{\infty}}^{X} f^{*}(V)$, and an application of Theorem 1 shows that $f$ is constant on a conjugacy invariant dense $G_{\delta}$ set. (In the above example of the point spectrum, it is well known that this fixed value is the empty set.) Similar remarks apply to $S_{1}(\mathbf{H})$.

(ii) Countable structures up to isomorphism. Assume that we assign to every $U \in U(\mathbf{H})$ a countable structure $f(U)$ of a fixed countable language $L$ (e.g., the language of fields, rings, groups, graphs, linear orderings, etc.) in such a way that the assignment in question constitutes a conjugacy invariant up to isomorphism, i.e. for any $U, V$ in $U(\mathbf{H}), U \approx$ $V \Rightarrow f(U) \cong f(V)$. Assume further that $f$ is nice, in the sense that there exists a Baire measurable mapping $f^{*}: U(\mathbf{H}) \rightarrow X_{L}\left(X_{L}\right.$ being the Polish space of all $L$-structures whose universe is $\mathbb{N}$, see, e.g., $[\mathbf{4}, 16 . \mathrm{C}])$ such that for any $U \in U(\mathbf{H}), f^{*}(U) \cong f(U)$. Then, if we consider the canonical logic action of $S_{\infty}$ on $X_{L}$ whose corresponding orbit equivalence relation is $\cong$ on $X_{L}$, we have that $U \approx V \Rightarrow f^{*}(U) \cong f^{*}(V)$, whenever $U, V$ are in $U(\mathbf{H})$. Therefore, by virtue of Theorem 1, there exists a conjugacy invariant dense $G_{\delta}$ subset of $U(\mathbf{H})$, for which $f(U)$ is fixed up to isomorphism.

The proof of Theorem 1 is reduced to the proof of the following related result concerning the Polish space $P(X)$ of all probability Borel measures on a perfect Polish space $X$ and the measure equivalence relation $\sim$.

THEOREM 2. Let $X$ be any perfect Polish space and let $Y$ be any Borel $S_{\infty}$-space. Then, for every Baire measurable function $f: P(X) \rightarrow Y$ with the property that $\mu \sim v \Rightarrow f(\mu) E_{S_{\infty}}^{Y} f(v)$, there exists a $\sim$-invariant dense $G_{\delta}$ subset $A$ of $P(X)$ such that $f[A]$ is contained in a single $E_{S_{\infty}}^{Y}$-class.

Remark. Again it should be pointed out that every measure class is meager (see, e.g., $\$ 4$ and the remark at the end of $\$ 5$ later).

Let us say that an equivalence relation $E$ on a Polish space $X$ is generically $S_{\infty}$-ergodic if every $E$-class is meager and for any Borel $S_{\infty}$-space $Y$ and for any Baire measurable function $f: X \rightarrow Y$ there is an $E$-invariant co-meager set $C \subseteq X$ such that $f[C]$ is contained in a single $E_{S_{\infty}}^{Y}$-class. In this terminology, Theorems 1 and 2 (and the remarks following them) assert that $\approx$ on $U(\mathbf{H}), S_{1}(\mathbf{H})$, and $\sim$ on $P(X)$ are generically $S_{\infty}$-ergodic.

The key tool in the proof of these theorems is Hjorth's theory of turbulence (see $[6,7])$.

Definition. Let $G$ be any Polish group acting continuously on a Polish space $X$ and let $x \in X$. For any open neighborhood $U$ of $x$ in $X$ and for any symmetric open neighborhood $V$ of $1^{G}$ in $G$, the $(U, V)$-local orbit $O(x, U, V)$ of $x$ in $X$ is defined as follows: $y \in O(x, U, V)$ if and only if there exist $g_{0}, g_{1}, \ldots, g_{k}$ in $V$ such that if $x_{0}=x$ and $x_{i+1}=g_{i} \cdot x_{i}$ for every $i \leq k$, then all the $x_{i}$ 's are in $U$ and $x_{k+1}=y$. The action of $G$ on $X$ is called turbulent at the point $x$, symbolically $x \in T_{G}^{X}$, if for any such $U$ and $V$ there exists an open neighborhood $U^{\prime}$ of $x$ in $X$ such that $U^{\prime} \subseteq U$ and $O(x, U, V)$ is dense in $U^{\prime}$. 
The concept of turbulence is a property of the orbits of the action in the sense that if $G$ is any Polish group acting continuously on a Polish space $X$ and $E_{G}^{X}$ stands for the corresponding orbit equivalence relation, then $T_{G}^{X}$ is $E_{G}^{X}$-invariant. The main result concerning the concept of turbulence that we will use is the following theorem of Hjorth [6].

THEOREM. (Hjorth). Let $G$ be any Polish group acting continuously on a Polish space $X$ in such a way that the orbits of the action are meager and at least one orbit is dense. Then the following are equivalent.

(i) The action of $G$ on $X$ is generically turbulent, in the sense that $T_{G}^{X}$ is co-meager in $X$.

(ii) $\quad\left(\exists x \in T_{G}^{X}\right)(\overline{G \cdot x}=X)$.

(iii) $E_{G}^{X}$ is generically $S_{\infty}$-ergodic, i.e. for any Borel $S_{\infty}$-space $Y$ and for any Baire measurable function $f: X \rightarrow Y$ with the property that $x E_{G}^{X} y \Rightarrow f(x) E_{S_{\infty}}^{Y} f(y)$, there exists an $E_{G}^{X}$-invariant co-meager set $A \subseteq X$ for which $f[A]$ is contained in a single $E_{S_{\infty}}^{Y}$-class.

(iv) The same as in (iii) but with 'Baire measurable' replaced by ' $C$-measurable' and 'co-meager' replaced by 'dense $G_{\delta}$ '. (Here $C$-measurable means measurable with respect to the smallest $\sigma$-algebra containing the open sets and closed under the Souslin operation.)

(v) For any Polish space $Y$ and for any Baire measurable function $f: X \rightarrow Y^{\mathbb{N}}$ with the property that $x E_{G}^{X} y \Rightarrow\{f(x)(n): n \in \mathbb{N}\}=\{f(y)(n): n \in \mathbb{N}\}$, there exists an $E_{G}^{X}$-invariant co-meager set $A \subseteq X$ and a countable set $\mathcal{C} \subseteq Y$ such that $x \in A \Rightarrow\{f(x)(n): n \in \mathbb{N}\}=\mathcal{C}$.

(vi) The same as in (v) but with 'Baire measurable' replaced by 'C-measurable' and 'co-meager' replaced by 'dense $G_{\delta}$ '.

(vii) For any countable language $L$ and for any Baire measurable function $f: X \rightarrow X_{L}$ with the property that $x E_{G}^{X} y \Rightarrow f(x) \cong f(y)$, there exists an $E_{G}^{X}$-invariant comeager subset $A$ of $X$ for which all countable structures in $f[A]$ are isomorphic.

(viii) The same as in (vii) but with 'Baire measurable' replaced by ' $C$-measurable' and 'co-meager' replaced by 'dense $G_{\delta}$ '.

Let us say that an equivalence relation $E$ on a Polish space $X$ admits classification by countable structures if there is a countable language $L$ and a Borel map $f: X \rightarrow X_{L}$ such that $x E y \Longleftrightarrow f(x) \cong f(y)$. Clearly if $E$ is generically $S_{\infty}$-ergodic, $E$ does not admit classification by countable structures, as $\cong$ on $X_{L}$ is induced by a Borel $S_{\infty}$-action. It is then a corollary of Theorem 2 that measure equivalence $\sim$ on $P(X)$, where $X$ is a perfect Polish space, does not admit classification by countable structures.

We give in $\S 2$ a different (and earlier) proof of this corollary using ideas from turbulence and a result of Kakutani in measure theory. In $\S 3$, we use similar ideas to give a new proof of the result of Preiss and Rataj [8], who, answering a question of Mauldin, showed that there is no maximal analytic set of pairwise orthogonal probability Borel measures on $[0,1]$. This means that for any analytic set $A$ of pairwise orthogonal measures, there exists a measure orthogonal to every measure in $A$. In fact, we prove the following strengthening, which shows that $\mu$ can be taken to be a product measure (if we switch to $2^{\mathbb{N}}$, instead of $[0,1])$. More precisely we have the following theorem. 
THEOREM 3. For any analytic set A of pairwise orthogonal probability Borel measures on the Cantor space $2^{\mathbb{N}}$, there exists $\alpha \in(0,1)^{\mathbb{N}}$ such that $\mu_{\alpha} \perp \mu$ for every $\mu \in A$, where $\mu_{\alpha}=\prod_{n \in \mathbb{N}}\left(\alpha(n) \delta_{0}+(1-\alpha(n)) \delta_{1}\right)$; hence no maximal set of pairwise orthogonal probability Borel measures on an uncountable Polish space can be analytic.

In $\S 4$, we review some facts concerning Baire category in the space of probability Borel measures. In $\S 5$, we prove Theorem 2 and in $\S 6$ we prove Theorem 1 .

Theorem 1 (for $U(\mathbf{H})$ ) and Theorem 2, as well as their proofs given here, were contained in a widely circulated preprint by the authors with the title 'A strong generic ergodicity property of unitary conjugacy'. Following that, Solecki [9] found another proof of Theorem 2 (for an arbitrary Polish space $X$ ), and Hjorth [10] found another proof of Theorem 1 for $U(\mathbf{H})$.

2. Non-classification of measure equivalence by countable structures

Recall that for equivalence relations $E, F$ on Polish spaces $X, Y$ we say that $E$ is Borel reducible to $Y$, in symbols $E \leq_{B} F$, if there is a Borel map $f: X \rightarrow Y$ such that $x_{1} E x_{2} \Longleftrightarrow f\left(x_{1}\right) F f\left(x_{2}\right)$.

THEOREM 2.1. Let $\sim$ stand for equivalence (i.e. mutual absolute continuity) of probability Borel measures on an uncountable Polish space. Then $\sim$ does not admit classification by countable structures.

Proof. We may as well assume that we work with the Cantor space. Identifying every $x \in \mathbb{Z}_{2}^{\mathbb{N}}$ with the corresponding subset of $\mathbb{N}$ of which it is the characteristic function, we have that $x+y=x \Delta y$, whenever $x, y$ are in $\mathbb{Z}_{2}^{\mathbb{N}}$, and $(\mathcal{P}(\mathbb{N}), \triangle)$ acquires the structure of a Polish group. Moreover, setting

$$
x \in I \Longleftrightarrow \sum_{n \in x} \frac{1}{n+1}<\infty,
$$

whenever $x \in \mathcal{P}(\mathbb{N})$, we obtain a Polishable subgroup $(I, \triangle)$ of $(\mathcal{P}(\mathbb{N}), \triangle)$ whose translation action on $\left(\mathcal{P}(\mathbb{N}), \triangle\right.$ ) is generically turbulent (see [6]) and consequently $E_{I}^{\mathcal{P}(\mathbb{N})}$ does not admit classification by countable structures. As the function $f: \mathcal{P}(\mathbb{N}) \rightarrow[0,1]^{\mathbb{N}}$ defined by

$$
f(x)(n)= \begin{cases}\frac{1}{\sqrt{n+1}}, & \text { if } n \in x, \\ 0, & \text { if } n \in \mathbb{N} \backslash x,\end{cases}
$$

whenever $x \in \mathcal{P}(\mathbb{N})$, is continuous and satisfies the condition

$$
|f(x)(n)-f(y)(n)|= \begin{cases}\frac{1}{\sqrt{n+1}}, & \text { if } n \in x \Delta y, \\ 0, & \text { if } n \in \mathbb{N} \backslash(x \triangle y),\end{cases}
$$

whenever $x, y$ are in $\mathcal{P}(\mathbb{N})$, it follows that

$$
x E_{I}^{\mathcal{P}(\mathbb{N})} y \Longleftrightarrow(f(x)(n)-f(y)(n))_{n \in \mathbb{N}} \in l_{2},
$$


hence $E_{I}^{\mathcal{P}(\mathbb{N})} \leq_{B} \sim_{2}$, where $u \sim_{2} v \Longleftrightarrow(u(n)-v(n))_{n \in \mathbb{N}} \in l_{2}$, whenever $u, v$ are in $[0,1]^{\mathbb{N}}$, and consequently it is enough to prove that $\sim_{2} \leq_{B} \sim$.

Setting $\mu_{\alpha}=\prod_{n \in \mathbb{N}}\left(\alpha(n) \delta_{0}+(1-\alpha(n)) \delta_{1}\right)$, whenever $\alpha \in[0,1]^{\mathbb{N}}$, we obtain a continuous function $[0,1]^{\mathbb{N}} \ni \alpha \mapsto \mu_{\alpha} \in P\left(\mathbb{Z}_{2}^{\mathbb{N}}\right)$. Moreover, by virtue of a theorem of Kakutani (see, e.g., $\left[11\right.$, p. 456]), if $\alpha, \beta$ in $[0,1]^{\mathbb{N}}$ are such that $(\exists \delta>0)(\forall n \in \mathbb{N})(\delta \leq$ $\alpha(n) \leq 1-\delta \wedge \delta \leq \beta(n) \leq 1-\delta)$, then

$$
\mu_{\alpha} \sim \mu_{\beta} \Longleftrightarrow \sum_{n=0}^{\infty}|\alpha(n)-\beta(n)|^{2}<\infty .
$$

Therefore, setting $f(x)(n)=(1+x(n)) / 4$, whenever $x \in[0,1]^{\mathbb{N}}$ and $n \in \mathbb{N}$, we obtain a continuous function $f:[0,1]^{\mathbb{N}} \rightarrow\left[\frac{1}{4}, \frac{1}{2}\right]^{\mathbb{N}}$ with the property that $x \sim_{2} y \Longleftrightarrow \mu_{f(x)} \sim$ $\mu_{f(y)}$, whenever $x, y$ are in $[0,1]^{\mathbb{N}}$.

Remark. Using Theorem 2.1 and the spectral theorem one can easily show that unitary equivalence of unitary and self-adjoint operators does not admit classification by countable structures. We do not provide the details here since they are similar to the arguments in $\S 6$ later.

3. An application: maximal sets of orthogonal measures

Mauldin et al. [12] raised the question of whether there exists a maximal set of pairwise orthogonal probability Borel measures on $[0,1]$, which is analytic. Preiss and Rataj [8] solved this problem by showing that they do not exist. We give below a different proof of this result, which actually provides some additional information.

THEOREM 3.1. For any analytic set A of pairwise orthogonal probability Borel measures on the Cantor space $2^{\mathbb{N}}$, there exists $\alpha \in(0,1)^{\mathbb{N}}$ such that $\mu_{\alpha} \perp \mu$ for every $\mu \in A$, where $\mu_{\alpha}=\prod_{n \in \mathbb{N}}\left(\alpha(n) \delta_{0}+(1-\alpha(n)) \delta_{1}\right)$; hence no maximal set of pairwise orthogonal probability Borel measures on an uncountable Polish space can be analytic.

We begin by giving the following definition.

Definition 3.2. Let $\preceq$ be an analytic partial pre-ordering on a Polish space $X$ and let stand for the associated equivalence relation, i.e.

$$
x \sim y \Longleftrightarrow(x \preceq y \wedge y \preceq x),
$$

whenever $x, y$ are in $X$, while $\perp$ stands for the associated orthogonality relation, i.e.

$$
x \perp y \Longleftrightarrow \neg(\exists z \in X)(z \preceq x \wedge z \preceq y),
$$

whenever $x, y$ are in $X$. Given $x \in X$, we shall say that $\perp$ satisfies the countable chain condition (ccc) below $x$ if the set $\{y \in X: y \preceq x\}$ does not contain uncountably many pairwise orthogonal elements.

We proceed by proving the following lemma.

LEMMA 3.3. Let $\preceq$ be an analytic partial pre-ordering on a Polish space $X$ such that $\perp$ satisfies the ccc below every $x \in X$ and assume that there exists a generically $S_{\infty}$-ergodic 
equivalence relation $E$ on a Polish space $Y$ and a Borel measurable function $f: Y \rightarrow X$ with the properties that $z E y \Rightarrow f(z) \sim f(y)$ and $\neg z E y \Rightarrow f(z) \perp f(y)$, whenever $z, y$ are in $Y$. Then, given any analytic subset $A$ of $X$, which consists of pairwise orthogonal elements, there exists $y \in Y$ such that $f(y) \perp x$ for every $x \in A$.

Proof. Towards a contradiction we assume the contrary, i.e. that $(\forall y \in Y)(\exists x \in$ $A)(\neg f(y) \perp x)$. Setting $G(y)=\{x \in A: \neg f(y) \perp x\}$ for every $y \in Y$, we obtain a countable non-empty subset of $A$ : indeed if $\left\{x_{i}: i \in I\right\} \subseteq G(y)$, with $i \neq j \Rightarrow x_{i} \neq x_{j}$, there is, for each $i \in I$, some $u_{i} \preceq x_{i}, u_{i} \preceq f(y)$. Since

$$
i \neq j \Rightarrow x_{i} \neq x_{j} \Rightarrow x_{i} \perp x_{j} \Rightarrow u_{i} \perp u_{j},
$$

and all $u_{i} \preceq f(y)$, we have that $I$ is countable, because $\perp$ satisfies the ccc below $f(y)$. Thus, the relation $R(y, x) \Longleftrightarrow x \in G(y)$, where $y \in Y$ and $x \in X$, is analytic with each section $R_{y}=G(y)$ countable and non-empty, which implies that there exists a $C$-measurable function $g: Y \rightarrow X^{\mathbb{N}}$ such that $\{g(y)(n): n \in \mathbb{N}\}=G(y)$ for every $y \in Y$. As $y E z \Rightarrow f(y) \sim f(z) \Rightarrow G(y)=G(z) \Rightarrow\{g(y)(n): n \in \mathbb{N}\}=\{g(z)(n): n \in \mathbb{N}\}$, whenever $y, z$ are in $Y$, the fact that $E$ is generically $S_{\infty}$-ergodic shows that there exists an $E$-invariant dense $G_{\delta}$ subset $A$ of $Y$ and a countable subset $\mathcal{C}$ of $X$ such that $y \in A \Rightarrow G(y)=\mathcal{C}$, whenever $y \in Y$. We will obtain a contradiction by showing that $A$ contains countably many $E$-equivalence classes and consequently it is meager: indeed if $\left\{y_{i}: i \in I\right\} \subseteq C$ and $i \neq j \Rightarrow \neg y_{i} E y_{j}$, then $i \neq j \Rightarrow f\left(y_{i}\right) \perp f\left(y_{j}\right)$. Fix $x \in a$. Then $x \in G\left(y_{i}\right)$, so there is $u_{i} \preceq x, u_{i} \preceq f\left(y_{i}\right)$. So $i \neq j \Rightarrow f\left(y_{i}\right) \perp f\left(y_{j}\right) \Rightarrow u_{i} \perp u_{j}$, thus, since $u_{i} \preceq x$, we have that $I$ is countable.

We are finally in a position to prove Theorem 3.1.

Proof of Theorem 3.1. Absolute continuity $\ll$ of probability Borel measures on the Cantor set constitutes a Borel partial pre-ordering on $P\left(2^{\mathbb{N}}\right)$ for which $\sim$, i.e. the associated equivalence relation, is mutual absolute continuity, and for which $\perp$, i.e. the associated orthogonality relation, is the usual orthogonality relation between measures. Since $\perp$ satisfies the ccc below every $\mu \in P\left(2^{\mathbb{N}}\right)$ (because if $v \ll \mu$ then $v \sim(\mu \mid A)$ for some Borel set $A \subseteq 2^{\mathbb{N}}$ of positive $\mu$-measure, so if $v \ll \mu$ and $\rho \ll \mu$ are orthogonal, then $v \sim(\mu \mid A), \rho \sim(\mu \mid B)$, and $\mu(A \cap B)=0)$, by virtue of the fact that the translation action of $(I, \triangle)$ on $(\mathcal{P}(\mathbb{N}), \triangle)$, as defined in $\S 2$, is generically turbulent, the preceding Lemma 3.3 shows that it is enough to construct a Borel measurable function $f: \mathcal{P}(\mathbb{N}) \rightarrow P\left(2^{\mathbb{N}}\right)$ with the properties that $z E_{I}^{\mathcal{P}(\mathbb{N})} y \Rightarrow f(z) \sim f(y)$ and $\neg z E_{I}^{\mathcal{P}(\mathbb{N})} y \Rightarrow f(z) \perp f(y)$, whenever $z, y$ are in $\mathcal{P}(\mathbb{N})$. To this end, setting

$$
\alpha(y)(n)= \begin{cases}\frac{1}{4}\left(1+\frac{1}{\sqrt{n+1}}\right), & \text { if } n \in y, \\ \frac{1}{4}, & \text { if } n \in \mathbb{N} \backslash y,\end{cases}
$$

for every $n \in \mathbb{N}$ and $f(y)=\mu_{\alpha(y)}$ for every $y \in \mathcal{P}(\mathbb{N})$, we obtain a continuous function $f: \mathcal{P}(\mathbb{N}) \rightarrow P\left(2^{\mathbb{N}}\right)$ such that if $z, y$ are in $\mathcal{P}(\mathbb{N})$, then

$$
\sum_{n=0}^{\infty}|\alpha(z)(n)-\alpha(y)(n)|^{2}=\sum_{n \in x \triangle y} \frac{1}{16(n+1)}
$$


and we need only appeal to the theorem of Kakutani mentioned in the proof of Theorem 2.1, which also shows that if

$$
\sum_{n=0}^{\infty}|\alpha(z)(n)-\alpha(y)(n)|^{2}=\infty
$$

then $\mu_{\alpha(z)} \perp \mu_{\alpha(y)}$.

\section{Baire category in the space of probability Borel measures}

The following result is well known but we provide a proof for the convenience of the reader (see also [1] for a proof in the case of the unit circle).

Proposition 4.1. If $X$ is any compact perfect Polish space, then $P_{c}(X)=\{\mu \in P(X)$ : $\mu$ is continuous, $P^{*}(X)=\{\mu \in P(X): \operatorname{supp}(\mu)=X\}$ and $v^{\perp}=\{\mu \in P(X): \mu \perp v\}$ constitute $\sim$-invariant dense $G_{\delta}$ 's in $P(X)$, while $\{\mu \in P(X): \mu \ll v$ and $d \mu / d \nu \in$ $\left.C\left(X, \mathbb{R}_{+}^{*}\right)\right\}$ is dense and meager in $P(X)$, whenever $v \in P^{*}(X)$.

Proof. We divide the argument into four steps.

(a) $P^{*}(X)$ constitutes a $\sim$-invariant dense $G_{\delta}$ in $P(X)$ : If $\left\{O_{n}: n \in \mathbb{N}\right\}$ is any countable basis for the topology on $X$, then $P^{*}(X)=\bigcap_{n \in \mathbb{N}}\left(\left\{\mu \in P(X): \mu\left(O_{n}\right)=0\right\}^{c}\right)$ and by virtue of the Baire category theorem and the Portmanteau Theorem (see, e.g., [4, 17.20]) it is enough to prove that for any non-empty open $O \subseteq X$, int $(\{\mu \in P(X): \mu(O)=0\})=\emptyset$. Towards a contradiction we assume the contrary and let $\lambda \in P(X),\left\{f_{0}, \ldots, f_{n}\right\} \subseteq$ $C(X, \mathbb{R})$ and $\epsilon>0$ be such that

$$
\left\{\mu \in P(X):(\forall i \leq n)\left(\left|\int_{X} f_{i} d \mu-\int_{X} f_{i} d \lambda\right|<\epsilon\right)\right\} \subseteq\{\mu \in P(X): \mu(O)=0\},
$$

while $x \in O$ and $\eta>0$ are such that

$$
\frac{\eta}{1+\eta} \max _{0 \leq i \leq n}\left(\left|\int_{X} f_{i} d \lambda\right|+\left|f_{i}(x)\right|\right)<\epsilon .
$$

Then

$$
\kappa=\frac{\lambda+\eta \delta_{x}}{1+\eta} \in P(X)
$$

and

$$
\kappa(O)=\frac{\eta}{1+\eta}>0
$$

while for any $0 \leq i \leq n$,

$$
\int_{X} f_{i} d \kappa-\int_{X} f_{i} d \lambda=\frac{\eta}{1+\eta}\left(\int_{X} f_{i} d \lambda+f_{i}(x)\right) \Rightarrow\left|\int_{X} f_{i} d \kappa-\int_{X} f_{i} d \lambda\right|<\epsilon,
$$

a contradiction.

(b) For any $v \in P(X), v^{\perp}$ constitutes a $\sim$-invariant dense $G_{\delta}$ in $P(X)$ : Since the set $\{x \in X: v(\{x\})>0\}$ is countable, if $\left\{O_{n}: n \in \mathbb{N}\right\}$ is any countable basis for the topology on $X$, an application of the Cantor-Bendixson Theorem shows that for any $n \in \mathbb{N}$, there exists $x_{n} \in O_{n}$ such that $v\left(\left\{x_{n}\right\}\right)=0$. Hence, $D=\left\{x_{n}: n \in \mathbb{N}\right\}$ is countable dense in $X$ and $v(D)=0$, which implies that $\{\mu \in P(X): \operatorname{supp}(\mu)$ is finite and contained in $D\} \subseteq v^{\perp}$ 
and consequently $v^{\perp}$ is dense in $P(X)$. In addition, if $\left\{c_{k}: k \in \mathbb{N}\right\}$ is any countable dense subset of $X$ and $\mathcal{B}=\left\{\bigcup_{i<n} B\left(c_{k_{i}} ; 2^{-l_{i}}\right):\left(k_{0}, \ldots, k_{n-1}\right),\left(l_{0}, \ldots, l_{n-1}\right) \in \mathbb{N}^{n}\right.$ and $\left.n \in \mathbb{N}\right\}$, then $v^{\perp}=\bigcap_{(m, n) \in \mathbb{N}^{2}} O_{m n}$, where for any $(m, n) \in \mathbb{N}^{2}$,

$$
O_{m n}=\bigcup_{B \in \mathcal{B} ; \nu(B)<2^{-(m+n)}}\left\{\mu \in P(X): \mu\left(B^{c}\right)<2^{-n}\right\},
$$

and consequently given any $(m, n) \in \mathbb{N}^{2}$, we need only prove that $O_{m n}$ is open in $P(X)$, which follows from the Portmanteau Theorem.

(c) $P_{c}(X)$ constitutes a $\sim$-invariant dense $G_{\delta}$ in $P(X)$ : We will first prove that $P_{c}(X)$ constitutes a $G_{\delta}$. Since $X$ is compact, both $P(X)$ and $K(X)$ are also compact and since $\operatorname{proj}_{P(X)}: P(X) \times X \ni(\mu, x) \mapsto \mu \in P(X)$ is obviously continuous, while $X \ni x \mapsto\{x\} \in K(X)$ constitutes an embedding, it follows that the function $P(X) \times X \ni(\mu, x) \mapsto \mu(\{x\}) \in[0,1]$ is upper semi-continuous and consequently its upper sections are closed and therefore compact, which implies that

$$
P(X) \backslash P_{c}(X)=\bigcup_{n \in \mathbb{N}} \operatorname{proj}_{P(X)}\left[\left\{(\mu, x) \in P(X) \times X: \mu(\{x\}) \geq 2^{-n}\right\}\right]
$$

is $K_{\sigma}$ in $P(X)$. The fact that $P_{c}(X)$ is dense in $P(X)$ will follow once we prove the fourth step of the argument, since $P_{c}(X) \cap P^{*}(X) \neq \emptyset$ and if $v \in P_{c}(X) \cap P^{*}(X)$, then

$$
\left\{\mu \in P(X): \mu \ll v \text { and } \frac{d \mu}{d \nu} \in C\left(X, \mathbb{R}_{+}^{*}\right)\right\} \subseteq P_{c}(X) \cap P^{*}(X) .
$$

(d) For any $v \in P^{*}(X),\left\{\mu \in P(X): \mu \ll v\right.$ and $\left.d \mu / d v \in C\left(X, \mathbb{R}_{+}^{*}\right)\right\}$ is dense and meager in $P(X)$ : By virtue of (b), it is enough to prove that the set in question is dense. To this end given $\kappa \in P(X)$ such that $\operatorname{supp}(\kappa)=\left\{x_{0}, \ldots, x_{l}\right\},\left\{f_{0}, \ldots, f_{n}\right\} \subseteq C(X, \mathbb{R})$ and $\epsilon>0$, we need only prove that there exists $\mu \in P(X)$ such that $\mu \ll v$ and $d \mu / d \nu \in C\left(X, \mathbb{R}_{+}^{*}\right)$, while for any $0 \leq i \leq n$,

$$
\left|\int_{X} f_{i} d \mu-\int_{X} f_{i} d \kappa\right|<\epsilon
$$

or (equivalently)

$$
\left|\int_{X} f_{i} d \mu-\sum_{k=0}^{l} \alpha_{k} f_{i}\left(x_{k}\right)\right|<\epsilon,
$$

where $\alpha_{k}=\kappa\left(\left\{x_{k}\right\}\right), 0 \leq k \leq l$. We set $M=\max _{0 \leq i \leq n}\left\|f_{i}\right\|_{\infty}$ and choose $\delta>0$ such that the balls $B\left(x_{k} ; \delta\right), 0 \leq k \leq l$, are pairwise disjoint and $\sup _{y \in B\left(x_{k} ; \delta\right)}\left|f_{i}(y)-f_{i}\left(x_{k}\right)\right|<\epsilon / 3$, for any pair of indices $i, k$. Given any $0 \leq k \leq l$, an application of the Urysohn Lemma for locally compact Hausdorff spaces shows that there exists a continuous function $\psi_{k}: X \rightarrow[0,1]$ such that $\psi_{k}=1$ in $\bar{B}\left(x_{k} ; \delta / 2\right)$ and $\psi_{k}=0$ out of $B\left(x_{k} ; \delta\right)$, and if $\beta_{k}=\int_{X} \psi_{k} d \nu$, then, since $\psi_{k}=1$ in $\bar{B}\left(x_{k} ; \delta / 2\right)$ and $\psi_{k}=0$ out of $B\left(x_{k} ; \delta\right)$, it follows that $\beta_{k} \geq v\left(B\left(x_{k} ; \delta / 2\right)\right)>0$ and $\beta_{k}=\int_{B\left(x_{k} ; \delta\right)} \psi_{k} d \nu$. We set $\beta=\min _{0 \leq k \leq l} \beta_{k}>0$ and let $0<\eta<1$ be such that $\eta(\eta+1)^{-1} M\left(\beta^{-1}+\eta\right)<\epsilon / 3$ and $\eta M<\epsilon / 3$. Setting $h=$ $\sum_{k=0}^{l} \alpha_{k} \beta_{k}^{-1} \psi_{k}+\eta$, it is not difficult to see that $\eta \leq h \leq \beta^{-1}+\eta, h: X \rightarrow\left[\eta, \beta^{-1}+\eta\right]$ 
is continuous and $\int_{X} h d v=1+\eta$. Therefore, if $d \mu=h(1+\eta)^{-1} d \nu$, then $\mu \ll v$ and $d \mu / d \nu \in C\left(X, \mathbb{R}_{+}^{*}\right)$. Moreover, given any $0 \leq i \leq n$,

$$
\begin{aligned}
\int_{X} f_{i} d \mu-\sum_{k=0}^{l} \alpha_{k} f_{i}\left(x_{k}\right)= & -\frac{\eta}{1+\eta} \int_{X} f_{i} h d v \\
& +\sum_{k=0}^{l} \alpha_{k} \int_{B\left(x_{k} ; \delta\right)}\left(f_{i}(y)-f_{i}\left(x_{k}\right)\right) \beta_{k}^{-1} \psi_{k}(y) d v(y) \\
& +\eta \int_{X} f_{i} d v
\end{aligned}
$$

Now

$$
\left|\int_{X} f_{i} h d \nu\right| \leq M\left(\beta^{-1}+\eta\right), \quad\left|\int_{X} f_{i} d \nu\right| \leq M
$$

and given $0 \leq k \leq l$,

$$
\left|\int_{B\left(x_{k} ; \delta\right)}\left(f_{i}(y)-f_{i}\left(x_{k}\right)\right) \beta_{k}^{-1} \psi_{k}(y) d \nu(y)\right| \leq \sup _{y \in B\left(x_{k} ; \delta\right)}\left|f_{i}(y)-f_{i}\left(x_{k}\right)\right|<\frac{\epsilon}{3},
$$

which implies that

$$
\left|\int_{X} f_{i} d \mu-\sum_{k=0}^{l} \alpha_{k} f_{i}\left(x_{k}\right)\right|<\frac{\eta}{1+\eta} M\left(\beta^{-1}+\eta\right)+\frac{\epsilon}{3} \sum_{k=0}^{l} \alpha_{k}+\eta M<\epsilon .
$$

5. Generic $S_{\infty}$-ergodicity for measures

THEOREM 5.1. If $X$ is any perfect Polish space and $\sim$ stands for equivalence of probability Borel measures on $X$, then $\sim$ is generically $S_{\infty}$-ergodic.

Notice that it is enough to prove Theorem 5.1 for compact perfect Polish spaces, by considering a compactification of $X$.

Indeed, since $X$ is homeomorphic to a $G_{\delta}$ subset of the Hilbert cube $[0,1]^{\mathbb{N}}$, the closure $\bar{X}$ of $X$ in $[0,1]^{\mathbb{N}}$ obviously constitutes a compact perfect Polish space and if $\left(O_{m}\right)_{m \in \mathbb{N}}$ is any descending sequence of open subsets of $\bar{X}$ with the property that $X=\bigcap_{m \in \mathbb{N}} O_{m}$, then it is enough to notice that

$$
P(X)=\bigcap_{(m, n) \in \mathbb{N}^{2}}\left\{\mu \in P(\bar{X}): \mu\left(O_{m}\right)>1-2^{-n}\right\}
$$

constitutes a dense $G_{\delta}$ in $P(\bar{X})$, something that follows from the fact that the $O_{m}$ 's are dense in $\bar{X}$ and the functions $P(\bar{X}) \ni \mu \mapsto \mu\left(O_{m}\right) \in[0,1], m \in \mathbb{N}$, are lower semicontinuous.

So for the rest of this section we will assume that $X$ is also compact. We begin by giving the following definitions.

Definition 5.2. We will say that an equivalence relation $E$ on a given Polish space $X$ admits an approximation by a Polish group action, when the following conditions are satisfied. 
(i) For any $x \in X$, there exists a Polish space $\Gamma_{x}$ and a continuous mapping $\phi_{x}: \Gamma_{x} \rightarrow$ $X$ such that $\phi_{x}\left[\Gamma_{x}\right]=[x]_{E}$.

(ii) There exists a Polish group $G$ acting continuously on $X$ with the property that, for any $x \in X$, there exists a continuous injection $i_{x}: G \hookrightarrow \Gamma_{x}$ such that $\overline{i_{x}[G]}=\Gamma_{x}$ and $\phi_{x}\left(i_{x}(g)\right)=g \cdot x$, whenever $g \in G$.

(iii) For any $x \in X$ and for any $\gamma \in \Gamma_{x}$, there exists a homeomorphism $\psi_{x, \gamma}: \Gamma_{x} \rightarrow$ $\Gamma_{\phi_{x}(\gamma)}$ with the property that $\phi_{x}(\delta)=\phi_{\phi_{x}(\gamma)}\left(\psi_{x, \gamma}(\delta)\right)$, whenever $\delta \in \Gamma_{x}$.

Definition 5.3. Let $E$ be any equivalence relation on a given Polish space $X$ and assume that it admits an approximation by a Polish group action. Then, keeping the same notation as in Definition 5.2, for any subset $A$ of $X$ its pseudo-Vaught transforms, $A^{*}$ and $A^{\triangle}$, are defined as follows:

$$
A^{*}=\left\{x \in X:\left(\forall^{*} \gamma \in \Gamma_{x}\right)\left(\phi_{x}(\gamma) \in A\right)\right\},
$$

and

$$
A^{\triangle}=\left\{x \in X:\left(\exists^{*} \gamma \in \Gamma_{x}\right)\left(\phi_{x}(\gamma) \in A\right)\right\}
$$

(Here $\forall^{*} \gamma$ means for 'co-meager many $\gamma$ ' and $\exists^{*} \gamma$ means 'for non-meager many $\gamma$. ')

The following proposition summarizes the basic properties of the pseudo-Vaught transforms.

Proposition 5.4. Let $E$ be any equivalence relation on a given Polish space $X$ and assume that it admits an approximation by a Polish group action. Then, keeping the same notation as in Definition 5.2, we have the following.

(a) The pseudo-Vaught transforms $P^{*}$ and $P^{\triangle}$ of any subset $P$ of $X$ are E-invariant and $(P)_{E} \subseteq P^{*} \subseteq P^{\triangle} \subseteq[P]_{E}$, where $(P)_{E}=\left\{x \in X:[x]_{E} \subseteq P\right\}$ and $[P]_{E}=\left\{x \in X:[x]_{E} \cap P \neq \emptyset\right\}$.

(b) For any $P \subseteq X, X \backslash P^{\triangle}=(X \backslash P)^{*}$ and $X \backslash P^{*}=(X \backslash P)^{\triangle}$.

(c) If $P, Q$ are any subsets of $X$, then $P \subseteq Q \Rightarrow\left(P^{\triangle} \subseteq Q^{\triangle} \wedge P^{*} \subseteq Q^{*}\right)$.

(d) If $P \subseteq X$ and $P_{n} \subseteq X$, whenever $n \in \mathbb{N}$, then $P=\bigcup_{n \in \mathbb{N}} P_{n} \Rightarrow P^{\triangle}=\bigcup_{n \in \mathbb{N}} P_{n}^{\triangle}$ and $P=\bigcap_{n \in \mathbb{N}} P_{n} \Rightarrow P^{*}=\bigcap_{n \in \mathbb{N}} P_{n}^{*}$.

(e) For any open $P \subseteq X, P^{*}$ constitutes $a G_{\delta}$.

(f) If $P \subseteq X$ is $E_{G}^{X}$-invariant and constitutes a $G_{\delta}$, then $P$ is contained in $P^{*}$. In particular, if $P \subseteq X$ is $E_{G}^{X}$-invariant and constitutes a dense $G_{\delta}$, then $P^{*}$ is $E$-invariant and constitutes a dense $G_{\delta}$.

Proof. Parts (b)-(d) are fairly straightforward and we will restrict ourselves to proving (a), (e) and (f).

(a) Since the fact that $(P)_{E} \subseteq P^{*} \subseteq P^{\triangle} \subseteq[P]_{E}$ is an immediate consequence of the definitions, we will restrict ourselves again to proving that both $P^{*}$ and $P^{\triangle}$ are $E$-invariant. Indeed, if $x \in P^{*}$ and $y \in P^{\triangle}$, while $\gamma \in \Gamma_{x}$ and $\delta \in \Gamma_{y}$, then $\left\{\alpha \in \Gamma_{x}: \phi_{x}(\alpha) \in P\right\}$ is co-meager in $\Gamma_{x}$ and $\left\{\beta \in \Gamma_{y}: \phi_{y}(\beta) \in P\right\}$ is non-meager in $\Gamma_{y}$, hence since the mappings $\psi_{x, \gamma}: \Gamma_{x} \rightarrow \Gamma_{\phi_{x}(\gamma)}$ and $\psi_{y, \delta}: \Gamma_{y} \rightarrow \Gamma_{\phi_{y}(\delta)}$ constitute homeomorphisms, while for any $\alpha \in \Gamma_{x}$ and for any $\beta \in \Gamma_{y}, \phi_{x}(\alpha)=\phi_{\phi_{x}(\gamma)}\left(\psi_{x, \gamma}(\alpha)\right)$ and $\phi_{y}(\beta)=\phi_{\phi_{y}(\delta)}\left(\psi_{y, \delta}(\beta)\right)$, 
it follows that

$$
\begin{aligned}
\psi_{x, \gamma}\left[\left\{\alpha \in \Gamma_{x}: \phi_{x}(\alpha) \in P\right\}\right] & =\left\{\psi_{x, \gamma}(\alpha): \alpha \in \Gamma_{x} \wedge \phi_{x}(\alpha) \in P\right\} \\
& =\left\{\psi_{x, \gamma}(\alpha): \alpha \in \Gamma_{x} \wedge \phi_{\phi_{x}(\gamma)}\left(\psi_{x, \gamma}(\alpha)\right) \in P\right\} \\
& =\left\{\alpha^{\prime} \in \Gamma_{\phi_{x}(\gamma)}: \phi_{\phi_{x}(\gamma)}\left(\alpha^{\prime}\right) \in P\right\}
\end{aligned}
$$

is co-meager in $\Gamma_{\phi_{x}(\gamma)}$ and consequently $\phi_{x}(\gamma) \in P^{*}$, while

$$
\begin{aligned}
\psi_{y, \delta}\left[\left\{\beta \in \Gamma_{y}: \phi_{y}(\beta) \in P\right\}\right] & =\left\{\psi_{y, \delta}(\beta): \beta \in \Gamma_{y} \wedge \phi_{y}(\beta) \in P\right\} \\
& =\left\{\psi_{y, \delta}(\beta): \beta \in \Gamma_{y} \wedge \phi_{\phi_{y}(\delta)}\left(\psi_{y, \delta}(\beta)\right) \in P\right\} \\
& =\left\{\beta^{\prime} \in \Gamma_{\phi_{y}(\delta)}: \phi_{\phi_{y}(\delta)}\left(\beta^{\prime}\right) \in P\right\}
\end{aligned}
$$

is non-meager in $\Gamma_{\phi_{y}(\delta)}$ and consequently $\phi_{y}(\delta) \in P^{\triangle}$.

(e) We choose at random a countable dense subset $C$ of $G$ and let $d$ be any complete compatible metric on $G$. Given $x \in X$, since the mapping $\phi_{x}: \Gamma_{x} \rightarrow X$ is continuous, $\left\{\gamma \in \Gamma_{x}: \phi_{x}(\gamma) \in P\right\}$ is open in $\Gamma_{x}$ and consequently it is co-meager in $\Gamma_{x}$ iff it is dense in $\Gamma_{x}$ or (equivalently) $(\forall n \in \mathbb{N})(\forall a \in C)(\exists b \in C)\left(d(a, b)<2^{-n} \wedge b \cdot x \in P\right)$, which is easily seen to imply that $P^{*}$ constitutes a $G_{\delta}$.

(f) If $x \in P$, then our assumptions imply that $i_{x}[G]$ is contained in $\left\{\gamma \in \Gamma_{x}\right.$ : $\left.\phi_{x}(\gamma) \in P\right\}$ which constitutes a $G_{\delta}$; therefore, $x \in P^{*}$.

The relation between the notion of generic $S_{\infty}$-ergodicity and the notion of approximation by a Polish group action for equivalence relations is demonstrated by the following proposition.

PROPOSITION 5.5. Every equivalence relation all of whose equivalence classes are meager and which admits an approximation by a generically turbulent Polish group action is generically $S_{\infty}$-ergodic.

Proof. Let $E$ be any equivalence relation on a given Polish space $X$ and assume that all its equivalence classes are meager and that it admits an approximation by a generically turbulent Polish group action. Then, keeping the same notation as in Definition 5.2, the fact that the action of $G$ on $X$ is generically turbulent implies that if $Y$ is any Borel $S_{\infty}$-space and $f: X \rightarrow Y$ is any $C$-measurable function with the property that $x E y \Rightarrow f(x) E_{S_{\infty}}^{Y} f(y)$, whenever $x, y$ are in $X$, then there exists an $E_{G}^{X}$-invariant dense $G_{\delta}$ subset $B$ of $X$ for which $f[B]$ is contained in a single $E_{S_{\infty}}^{Y}$-class. Thus, setting $A=B^{*}$, Proposition 5.4 is easily seen to imply that $A$ constitutes an $E$-invariant dense $G_{\delta}$ subset of $X$ for which $f[A]$ is contained in a single $E_{S_{\infty}}^{Y}$-class.

Proposition 4.1 is easily seen to imply that every -equivalence class is meager and consequently, by virtue of Proposition 5.5, in order to prove Theorem 5.1 it is enough to show that mutual absolute continuity admits an approximation by a generically turbulent Polish group action.

Proposition 5.6. If $X$ is any compact perfect Polish space, then we have the following.

(i) For any $\mu \in P(X), L_{++}^{1}(X, \mu)=\left\{f \in L^{1}(X, \mu): f>0, \mu\right.$-a.e. $\}$ constitutes a dense $G_{\delta}$ in $L_{+}^{1}(X, \mu)=\left\{f \in L^{1}(X, \mu): f \geq 0\right.$, $\mu$-a.e. $\}$, and the mapping $\Phi_{\mu}$ : $L_{+}^{1}(X, \mu) \backslash\{0\} \rightarrow P(X)$ defined by the relation $d\left(\Phi_{\mu}(f)\right)=\left(\int_{X} f d \mu\right)^{-1} f d \mu$ is continuous and satisfies the condition $\Phi_{\mu}\left[L_{++}^{1}(X, \mu)\right]=[\mu]_{\sim}$. 
(ii) $C\left(X, \mathbb{R}_{+}^{*}\right)=\{f: X \rightarrow \mathbb{R}: f$ is continuous and $f>0\}$ constitutes a group under pointwise multiplication, the group structure in question being compatible with the topology of uniform convergence which is Polish on $C\left(X, \mathbb{R}_{+}^{*}\right)$, and it acts continuously on $P(X)$ via $C\left(X, \mathbb{R}_{+}^{*}\right) \times P(X) \ni(f, \mu) \mapsto \Phi_{\mu}(f) \in P(X)$. Also for any $\mu \in P(X), \overline{C\left(X, \mathbb{R}_{+}^{*}\right)}=L_{++}^{1}(X, \mu)$ (where we identify here any $f \in C\left(X, \mathbb{R}_{+}^{*}\right)$ with the unique element of $L_{++}^{1}(X, \mu)$ containing $\left.f\right)$.

(iii) For any $\mu \in P(X)$ and for any $f \in L_{++}^{1}(X, \mu)$, the mapping $\Psi_{\mu, f}: L_{++}^{1}(X, \mu) \ni$ $g \mapsto g\left(\int_{X} f d \mu\right) / f \in L_{++}^{1}\left(X, \Phi_{\mu}(f)\right)$ constitutes a homeomorphism with the property that $\Phi_{\mu}(g)=\Phi_{\Phi_{\mu}(f)}\left(\Psi_{\mu, f}(g)\right)$.

Proof. (i) We divide the argument into two steps.

(a) $L_{++}^{1}(X, \mu)$ constitutes a dense $G_{\delta}$ in $L_{+}^{1}(X, \mu)$ : If $\epsilon, \delta$ are arbitrary positive rationals, then we set $H_{\epsilon}^{(\delta)}=\left\{f \in L_{+}^{1}(X, \mu): \mu(\{x \in X: f(x)>\epsilon\})>1-\delta\right\}$ and let $H=\bigcap_{\delta \in \mathbf{Q}_{+}^{*}} \bigcup_{\epsilon \in \mathbf{Q}_{+}^{*}} H_{\epsilon}^{(\delta)}$. It is not difficult to verify that $H=L_{++}^{1}(X, \mu)$ and consequently we need only prove that, for any $\delta \in \mathbf{Q}_{+}^{*}$, the set $H^{(\delta)}=\bigcup_{\epsilon \in \mathbf{Q}_{+}^{*}} H_{\epsilon}^{(\delta)}$ is open and dense in $L_{+}^{1}(X, \mu)$.

We shall first prove that $H^{(\delta)}$ is open in $L_{+}^{1}(X, \mu)$. So let $f \in H^{(\delta)}$ and let $\epsilon \in \mathbf{Q}_{+}^{*}$ be such that $\mu(\{x \in X: f(x)>\epsilon\})>1-\delta$. We set $\eta=\mu(\{x \in X: f(x)>\epsilon\})-(1-\delta)>0$ and let $g$ be any non-negative function in $L^{1}(X, \mu)$ such that $\int_{X}|f-g| d \mu<\epsilon \eta / 2$. If

$$
E=\left\{x \in X:|f(x)-g(x)|>\frac{\epsilon}{2}\right\}
$$

then obviously

$$
\frac{\epsilon}{2} \mu(E) \leq \int_{E}|f(x)-g(x)| d \mu(x)<\frac{\epsilon \eta}{2}
$$

and hence

$$
\mu\left(\left\{x \in X:|f(x)-g(x)|>\frac{\epsilon}{2}\right\}\right)<\eta
$$

Thus, since

$$
\left\{x \in X: g(x) \leq \frac{\epsilon}{2}\right\} \cap\{x \in X: f(x)>\epsilon\} \subseteq\left\{x \in X:|f(x)-g(x)|>\frac{\epsilon}{2}\right\}
$$

and hence

$$
\left\{x \in X: g(x) \leq \frac{\epsilon}{2}\right\} \subseteq\{x \in X: f(x) \leq \epsilon\} \cup\left\{x \in X:|f(x)-g(x)|>\frac{\epsilon}{2}\right\},
$$

it follows that

$$
\begin{aligned}
\mu\left(\left\{x \in X: g(x) \leq \frac{\epsilon}{2}\right\}\right) \leq & \mu(\{x \in X: f(x) \leq \epsilon\}) \\
& +\mu\left(\left\{x \in X:|f(x)-g(x)|>\frac{\epsilon}{2}\right\}\right) \\
< & 1-\mu(\{x \in X: f(x)>\epsilon\})+\eta=\delta
\end{aligned}
$$

and consequently

$$
\mu\left(\left\{x \in X: g(x)>\frac{\epsilon}{2}\right\}\right)>1-\delta
$$


i.e. $g \in H_{\epsilon / 2}^{(\delta)}$. We have thus proved that

$$
\left\{g \in L_{+}^{1}(X, \mu): \int_{X}|f-g| d \mu<\frac{\epsilon \eta}{2}\right\} \subseteq H^{(\delta)}
$$

and consequently $H^{(\delta)}$ is open in $L_{+}^{1}(X, \mu)$.

What is left to show is that $H^{(\delta)}$ is dense in $L_{+}^{1}(X, \mu)$. So let $f$ be any non-negative function in $L^{1}(X, \mu)$ and let $\epsilon \in \mathbf{Q}_{+}^{*}$. Then evidently $f+(\epsilon / 2) \in L_{++}^{1}(X, \mu)$ and $f+(\epsilon / 2) \in H^{(\delta)}$.

(b) $\Phi_{\mu}: L_{+}^{1}(X, \mu) \backslash\{0\} \rightarrow P(X)$ is continuous and satisfies the condition $\Phi_{\mu}\left[L_{++}^{1}(X, \mu)\right]=[\mu]_{\sim}:$ If $f_{n} \rightarrow f$ in $L_{+}^{1}(X, \mu) \backslash\{0\}$ as $n \rightarrow \infty$, then evidently

$$
\left|\int_{X} f_{n} d \mu-\int_{X} f d \mu\right| \leq \int_{X}\left|f_{n}-f\right| d \mu \rightarrow 0
$$

as $n \rightarrow \infty$ and for any $g \in C(X, \mathbb{R})$,

$$
\left|\int_{X} g f_{n} d \mu-\int_{X} g f d \mu\right| \leq\|g\|_{\infty} \cdot \int_{X}\left|f_{n}-f\right| d \mu \rightarrow 0
$$

as $n \rightarrow \infty$. Therefore,

$$
\begin{aligned}
\int_{X} g d\left(\Phi_{\mu}\left(f_{n}\right)\right) & =\int_{X} g f_{n} d \mu \cdot\left(\int_{X} f_{n} d \mu\right)^{-1} \rightarrow \int_{X} g f d \mu \cdot\left(\int_{X} f d \mu\right)^{-1} \\
& =\int_{X} g d\left(\Phi_{\mu}(f)\right)
\end{aligned}
$$

as $n \rightarrow \infty$, whenever $g \in C(X, \mathbb{R})$, and consequently $\Phi_{\mu}\left(f_{n}\right) \rightarrow \Phi_{\mu}(f)$ in $P(X)$ as $n \rightarrow \infty$, which implies that the mapping $\Phi_{\mu}: L_{+}^{1}(X, \mu) \backslash\{0\} \rightarrow P(X)$ is continuous. The fact that it satisfies the condition $\Phi_{\mu}\left[L_{++}^{1}(X, \mu)\right]=[\mu] \sim$ follows immediately from the Radon-Nikodym Theorem.

(ii) It is straightforward to verify that $C\left(X, \mathbb{R}_{+}^{*}\right) \times P(X) \ni(f, \mu) \mapsto \Phi_{\mu}(f) \in P(X)$ constitutes an action whose continuity follows from part (i), while the density of $C\left(X, \mathbb{R}_{+}^{*}\right)$ in $L_{++}^{1}(X, \mu)$ follows from Lusin's Theorem (see Rudin [13, 2.23]).

(iii) This is straightforward.

Thus, by virtue of Propositions 5.5 and 5.6, in order to complete the proof of Theorem 5.1, it is enough to show that the action of $C\left(X, \mathbb{R}_{+}^{*}\right)$ on $P(X)$ described in Proposition 5.6 is generically turbulent. The following lemma establishes a sufficient condition for turbulence.

Lemma 5.7. Let $G$ be any Polish group acting continuously on a Polish space $X$ and let $x \in X$. Suppose $G \cdot x$ is dense in $X$ and there exists a fundamental system of open neighborhoods $U$ of $x$ in $X$ with the property that, for any $g \in G$ for which $g \cdot x \in U$, there exists $h \in G$ and a continuous path $[0,1] \ni t \mapsto h_{t} \in G$ such that $g \cdot x=h \cdot x$, $h_{0}=1^{G}, h_{1}=h$ and $h_{t} \cdot x \in U$, whenever $t \in[0,1]$. Then the action of $G$ on $X$ is turbulent at the point $x$.

Proof. Let $V$ be any open neighborhood of $x$ in $X$ and let $W$ be any symmetric open neighborhood of the identity in $G$. Then there exists an open neighborhood $U$ of $x$ in 
$X$ which is contained in $V$ and satisfies the condition stated in the formulation of the lemma. We need only prove that $O(x, U, W)=U \cap(G \cdot x)$. So let $g \in G$ be such that $g \cdot x \in U \cap(G \cdot x)$ and let $h \in G$ and [0,1] $\ni t \mapsto h_{t} \in G$ be as in the statement of the lemma. Then there exists a positive integer $N$ such that for any $s, t$ in $[0,1],|s-t| \leq N^{-1} \Rightarrow h_{s} \cdot h_{t}^{-1} \in W$. Hence, setting $t_{0}=0, t_{k}=t_{k-1}+N^{-1}$ and $g_{k}=h_{t_{k}} \cdot h_{t_{k-1}}^{-1}$, whenever $1 \leq k \leq N$, it follows immediately that $g_{k} \in W$ and $g_{k} \ldots g_{1} \cdot x=h_{t_{k}} \cdot x \in U$, whenever $1 \leq k \leq N$, while $g_{N} \ldots g_{1} \cdot x=g \cdot x$. We have thus proved that $O(x, U, W)=U \cap(G \cdot x)$.

Thus, by virtue of Proposition 4.1 and Lemma 5.7, in order to prove that the action of $C\left(X, \mathbb{R}_{+}^{*}\right)$ on $P(X)$, described in Proposition 5.6, is generically turbulent, it is enough to verify that the system of open neighborhoods of $\mu \in P^{*}(X)$, which consists of the sets of the form

$$
U_{\mu ; f_{0}, \ldots, f_{n} ; \epsilon}=\left\{\nu \in P(X):(\forall i \leq n)\left(\left|\int_{X} f_{i} d \nu-\int_{X} f_{i} d \mu\right|<\epsilon\right)\right\},
$$

where $\left\{f_{0}, \ldots, f_{n}\right\} \subseteq C(X, \mathbb{R})$ and $\epsilon>0$, satisfies the condition stated in the formulation of Lemma 5.7. Indeed, if $g$ is any function in the group considered, such that $g \cdot \mu \in$ $U_{\mu ; f_{0}, \ldots, f_{n} ; \epsilon}$, then we need only take $h=\left(\int_{X} g d \mu\right)^{-1} \cdot g$ and $h_{t}=(1-t)+t h$, whenever $t \in[0,1]$.

\section{Generic turbulence for unitary and self-adjoint operators}

THEOREM 6.1. Let $\mathbf{H}$ be any infinite-dimensional separable complex Hilbert space and let $U(\mathbf{H})$ stand for the Polish group of unitary operators on $\mathbf{H}$, while $S_{1}(\mathbf{H})$ stands for the Polish space of self-adjoint operators on $\mathbf{H}$ with norm at most 1 , both equipped with the strong topology. Then the conjugation action of $U(\mathbf{H})$ on both $U(\mathbf{H})$ and $S_{1}(\mathbf{H})$ is generically turbulent.

We begin by giving the following definition.

Definition 6.2. When a complete orthonormal system $\left\{\mathbf{e}_{n}: n \in \mathbb{N}\right\}$ in $\mathbf{H}$ is fixed, a canonical representative of its maximal spectral type is assigned to every normal bounded linear operator $T$ on $\mathbf{H}$ as follows:

$$
\mu_{T}(B)=\sum_{n=0}^{\infty} 2^{-(n+1)} E_{\mathbf{e}_{n}, \mathbf{e}_{n}}^{T}(B) \quad(B \in \mathbf{B}(\sigma(T))),
$$

where $E^{T}$ denotes the spectral measure and $\sigma(T)$ the spectrum that correspond to $T$ and $E_{x, y}^{T}(B)=\left(E^{T}(B) x, y\right)(B \in \mathbf{B}(\sigma(T)))$ for every $x, y$ in $\mathbf{H}$.

The importance of the canonical representative of the maximal spectral type of a normal bounded linear operator on $\mathbf{H}$ is demonstrated by the following proposition.

Proposition 6.3. If $T$ and $T_{n}, n \in \mathbb{N}$, are any normal bounded linear operators on $\mathbf{H}$ whose spectrum is contained in $K \in K(\mathbb{C}) \backslash\{\emptyset\}$ and $T_{n} \rightarrow T$ in $L(\mathbf{H})$ as $n \rightarrow \infty$, with respect to the strong topology, then $\mu_{T_{n}} \rightarrow \mu_{T}$ in $P(K)$ as $n \rightarrow \infty$. 
Proof. We will first prove the following standard claim: For any continuous function $f: K \rightarrow \mathbb{C}, f\left(T_{n}\right) \rightarrow f(T)$ in $L(\mathbf{H})$ as $n \rightarrow \infty$, with respect to the strong topology.

Let $u, v$ be any unit vectors on $\mathbf{H}$ and let $p(z) \in \mathbb{C}[z]$. Then, the proof of the spectral theorem shows that

$\left(\left(f\left(T_{n}\right)-f(T)\right) u, v\right)=\int_{K}(f-p) d E_{u, v}^{T_{n}}+\left(\left(p\left(T_{n}\right)-p(T)\right) u, v\right)+\int_{K}(p-f) d E_{u, v}^{T}$,

so

$$
\begin{aligned}
\left|\left(\left(f\left(T_{n}\right)-f(T)\right) u, v\right)\right| \leq & \int_{K}|f-p|\left|d E_{u, v}^{T_{n}}\right|+\left|\left(\left(p\left(T_{n}\right)-p(T)\right) u, v\right)\right| \\
& +\int_{K}\left|f-p\left\|d E_{u, v}^{T} \mid \leq 2 \cdot\right\| f-p\left\|_{\infty} \cdot\right\| u\|\cdot\| v \|\right. \\
& +\left|\left(\left(p\left(T_{n}\right)-p(T)\right) u, v\right)\right|,
\end{aligned}
$$

for any $n \in \mathbb{N}$, and consequently

$$
\begin{aligned}
\left\|\left(f\left(T_{n}\right)-f(T)\right) u\right\| & =\sup _{\|v\|=1}\left|\left(\left(f\left(T_{n}\right)-f(T)\right) u, v\right)\right| \\
& \leq 2 \cdot\|f-p\|_{\infty} \cdot\|u\|+\sup _{\|v\|=1}\left|\left(\left(p\left(T_{n}\right)-p(T)\right) u, v\right)\right| \\
& =2 \cdot\|f-p\|_{\infty} \cdot\|u\|+\left\|\left(p\left(T_{n}\right)-p(T)\right) u\right\|
\end{aligned}
$$

for every $n \in \mathbb{N}$. Hence, by virtue of the Stone-Weierstass Theorem, it is enough to prove that $\left\|\left(p\left(T_{n}\right)-p(T)\right) u\right\| \rightarrow 0$ as $n \rightarrow \infty$. Indeed, if $p(z)$ has degree $N$ and

$$
p(z)=\sum_{k=0}^{N} \alpha_{k} z^{k},
$$

where the $\alpha_{k}$ 's are in $\mathbb{C}$ and $\alpha_{N} \neq 0$, then the proof of the spectral theorem shows that

$$
\begin{aligned}
\left\|\left(p\left(T_{n}\right)-p(T)\right) u\right\| & \leq \sum_{k=0}^{N}\left|\alpha_{k}\right| \cdot\left\|\left(T_{n}^{k}-T^{k}\right) u\right\| \\
& \leq \sum_{k=0}^{N}\left|\alpha_{k}\right| \cdot k \cdot\left(\sup _{z \in K}|z|\right)^{k-1} \cdot\left\|\left(T_{n}-T\right) u\right\| \rightarrow 0 \quad \text { as } n \rightarrow \infty .
\end{aligned}
$$

Now to prove the proposition, by virtue of the Portmanteau Theorem, given any closed $F \subseteq K$, it is enough to show that

$$
\limsup _{n \rightarrow \infty} \mu_{T_{n}}(F) \leq \mu_{T}(F)
$$

and since for any $n, N \in \mathbb{N}$,

$$
\mu_{T_{n}}(F) \leq \sum_{k=0}^{N} 2^{-(k+1)} E_{\mathbf{e}_{k}, \mathbf{e}_{k}}^{T_{n}}(F)+\sum_{k>N} 2^{-(k+1)},
$$

it is enough to prove that for any $k \in \mathbb{N}$,

$$
\limsup _{n \rightarrow \infty} E_{\mathbf{e}_{k}, \mathbf{e}_{k}}^{T_{n}}(F) \leq E_{\mathbf{e}_{k}, \mathbf{e}_{k}}^{T}(F),
$$


whenever $F \subseteq K$ is closed, or (equivalently) that $E_{\mathbf{e}_{k}, \mathbf{e}_{k}}^{T_{n}} \rightarrow E_{\mathbf{e}_{k}, \mathbf{e}_{k}}^{T}$ in $P(K)$ as $n \rightarrow \infty$; but this follows from the above-mentioned claim, since for any $f \in C(K, \mathbb{C})$,

$$
\begin{aligned}
\left|\int_{K} f d E_{\mathbf{e}_{k}, \mathbf{e}_{k}}^{T_{n}}-\int_{K} f d E_{\mathbf{e}_{k}, \mathbf{e}_{k}}^{T}\right| & =\left|\left(\left(f\left(T_{n}\right)-f(T)\right) \mathbf{e}_{k}, \mathbf{e}_{k}\right)\right| \\
& \leq\left\|\left(f\left(T_{n}\right)-f(T)\right) \mathbf{e}_{k}\right\| \rightarrow 0 \quad \text { as } n \rightarrow \infty
\end{aligned}
$$

Thus, since the functions that assign a measure in $P(\mathbf{T})$ to every operator in $U(\mathbf{H})$ and a measure in $P([-1,1])$ to every operator in $S_{1}(\mathbf{H})$ as a canonical representative of its maximal spectral type are continuous, we will reduce the proof of Theorem 6.1 to Theorem 5.1. To this end, we will first prove that there exist Borel inverses of the functions mentioned above that assign an operator in $U(\mathbf{H})$ to every measure in $P^{*}(\mathbf{T}) \cap P_{c}(\mathbf{T})$ and an operator in $S_{1}(\mathbf{H})$ to every measure in $P^{*}([-1,1]) \cap P_{c}([-1,1])$, and we will then prove that the Polish group actions considered in Theorem 6.1 are generically turbulent by proving that they satisfy the antecedents and part (iv) of the succedants of the theorem of Hjorth mentioned in $\S 1$.

Definition 6.4. Given $\mu \in P^{*}(\mathbf{T}) \cap P_{c}(\mathbf{T})$ and $\nu \in P^{*}([-1,1]) \cap P_{c}([-1,1])$, let $f_{\mu}$ stand for the function $e^{2 \pi i t} \mapsto \mu\left(\left\{e^{2 \pi i s}: 0 \leq s<t\right\}\right) \bmod 1(t \in[0,1))$ and let $g_{v}$ stand for the function $x \mapsto v([-1, x])(x \in[-1,1])$.

LEMMA 6.5. For any $\mu \in P^{*}(\mathbf{T}) \cap P_{c}(\mathbf{T})$ and for any $v \in P^{*}([-1,1]) \cap P_{c}([-1,1])$, the mappings $\Phi_{\mu}: L^{2}\left([0,1), m_{1}\right) \ni f \mapsto f \circ f_{\mu} \in L^{2}(\mathbf{T}, \mu)$ and $\Psi_{v}: L^{2}\left([0,1], m_{1}\right) \ni$ $g \mapsto g \circ g_{v} \in L^{2}([-1,1], v)$ constitute Hilbert space isomorphisms.

Proof. Straightforward.

LEMMA 6.6. Given $\kappa \in P^{*}(\mathbf{T}) \cap P_{c}(\mathbf{T})$ the mapping $U_{\kappa} f=f_{\kappa}^{-1} \cdot f\left(f \in L^{2}\left([0,1), m_{1}\right)\right)$ constitutes a multiplicity-free unitary operator on $L^{2}\left([0,1), m_{1}\right)$, with $\mu_{U_{\kappa}}=\kappa$ with respect to the standard basis in $L^{2}\left([0,1), m_{1}\right)$, which consists of the functions $e_{n}$ : $x \mapsto e^{2 \pi i n x}(x \in[0,1) ; n \in \mathbb{Z})$. Given $\lambda \in P^{*}([-1,1]) \cap P_{c}([-1,1])$ the mapping $S_{\lambda} g=g_{\lambda}^{-1} \cdot g\left(g \in L^{2}\left([0,1], m_{1}\right)\right)$ constitutes a multiplicity-free self-adjoint operator on $L^{2}\left([0,1], m_{1}\right)$ with norm at most 1 , and with $\mu_{S_{\lambda}}=\lambda$ with respect to the standard basis in $L^{2}\left([0,1], m_{1}\right)$, which consists of the functions $e_{n}: x \mapsto e^{2 \pi i n x}(x \in[0,1] ; n \in \mathbb{Z})$.

Proof. For any $f \in L^{2}\left([0,1), m_{1}\right)$ and for any $g, h \in L^{2}\left([0,1], m_{1}\right)$,

$$
\begin{aligned}
& \left(\left\|U_{\kappa} f\right\|_{2}\right)^{2}=\int_{0}^{1}\left|f_{\kappa}^{-1} \cdot f\right|^{2} d m_{1}=\int_{0}^{1}\left|f_{\kappa}^{-1}\right|^{2} \cdot|f|^{2} d m_{1}=\left(\|f\|_{2}\right)^{2}, \\
& \left(\left\|S_{\lambda} g\right\|_{2}\right)^{2}=\int_{0}^{1}\left|g_{\lambda}^{-1} \cdot g\right|^{2} d m_{1} \leq\left(\left\|g_{\lambda}^{-1}\right\|_{\infty}\right)^{2} \cdot \int_{0}^{1}|g|^{2} d m_{1} \leq\left(\|g\|_{2}\right)^{2}
\end{aligned}
$$

and

$$
\left(S_{\lambda} g, h\right)=\int_{0}^{1} g_{\lambda}^{-1} g \cdot \bar{h} \cdot d m_{1}=\int_{0}^{1} g \cdot \overline{g_{\lambda}^{-1} h} \cdot d m_{1}=\left(g, S_{\lambda} h\right),
$$


which implies that $U_{\kappa} \in U\left(L^{2}\left([0,1), m_{1}\right)\right)$ and $S_{\lambda} \in S_{1}\left(L^{2}\left([0,1], m_{1}\right)\right)$. Moreover, if $f \in L^{2}(\mathbf{T}, \kappa)$ and $g \in L^{2}([-1,1], \lambda)$, then

$$
\begin{aligned}
\left(\Phi_{\kappa} U_{\kappa} \Phi_{\kappa}^{-1} f\right)(\zeta) & =\left(\Phi_{\kappa} U_{\kappa}\left(f \circ f_{\kappa}^{-1}\right)\right)(\zeta)=\left(\Phi_{\kappa}\left(f_{\kappa}^{-1} \cdot\left(f \circ f_{\kappa}^{-1}\right)\right)\right)(\zeta) \\
& =\left(\left(f_{\kappa}^{-1} \circ f_{\kappa}\right) \cdot\left(\left(f \circ f_{\kappa}^{-1}\right) \circ f_{\kappa}\right)\right)(\zeta)=\zeta \cdot f(\zeta),
\end{aligned}
$$

whenever $\zeta \in \mathbf{T}$, and similarly $\left(\Psi_{\lambda} S_{\lambda} \Psi_{\lambda}^{-1} g\right)(x)=x \cdot g(x)$, whenever $x \in[-1,1]$, which implies that $\Phi_{\kappa} U_{\kappa} \Phi_{\kappa}^{-1}$ is multiplicity-free and

$$
E^{\Phi_{\kappa} U_{\kappa} \Phi_{\kappa}^{-1}}(B) f=\chi_{B} \cdot f\left(B \in \mathbf{B}(\mathbf{T}) ; f \in L^{2}(\mathbf{T}, \kappa)\right),
$$

while $\Psi_{\lambda} S_{\lambda} \Psi_{\lambda}^{-1}$ is multiplicity-free and

$$
E^{\Psi_{\lambda} S_{\lambda} \Psi_{\lambda}^{-1}}(B) g=\chi_{B} \cdot g\left(B \in \mathbf{B}([-1,1]) ; g \in L^{2}([-1,1], \lambda)\right) .
$$

Therefore, we deduce that $U_{\kappa}$ is multiplicity-free and

$$
E^{U_{\kappa}}(B)=\Phi_{\kappa}^{-1} E^{\Phi_{\kappa} U_{\kappa} \Phi_{\kappa}^{-1}}(B) \Phi_{\kappa}(B \in \mathbf{B}(\mathbf{T})),
$$

while $S_{\lambda}$ is multiplicity-free and

$$
E^{S_{\lambda}}(B)=\Psi_{\lambda}^{-1} E^{\Psi_{\lambda} S_{\lambda} \Psi_{\lambda}^{-1}}(B) \Psi_{\lambda}(B \in \mathbf{B}([-1,1])) .
$$

Hence

$$
\begin{aligned}
E_{e_{n}, e_{n}}^{U_{\kappa}}(B) & =\left(E^{U_{\kappa}}(B) e_{n}, e_{n}\right)=\left(E^{\Phi_{\kappa} U_{\kappa} \Phi_{\kappa}^{-1}}(B) \Phi_{\kappa} e_{n}, \Phi_{\kappa} e_{n}\right) \\
& =\int_{\mathbf{T}} \chi_{B} \cdot\left(e_{n} \circ f_{\kappa}\right) \cdot \overline{\left(e_{n} \circ f_{\kappa}\right)} d \kappa=\int_{B}\left|e_{n} \circ f_{\kappa}\right|^{2} d \kappa=\kappa(B),
\end{aligned}
$$

whenever $B \in \mathbf{B}(\mathbf{T})$, and similarly $E_{e_{n}, e_{n}}^{S_{\lambda}}(B)=\lambda(B)$, whenever $B \in \mathbf{B}([-1,1])$, which implies that $E_{e_{n}, e_{n}}^{U_{\kappa}}=\kappa$ and $E_{e_{n}, e_{n}}^{S_{\lambda}}=\lambda$, whenever $n \in \mathbb{Z}$ and consequently $\mu_{U_{\kappa}}=\kappa$ and $\mu_{S_{\lambda}}=\lambda$.

In the following denote by $V \cdot U=V U V^{-1}$ the conjugacy action of $U(\mathbf{H})$ on $U(\mathbf{H})$ or $S_{1}(\mathbf{H})$, and by $\approx$ the unitary conjugacy equivalence relation. We also let

$$
\begin{gathered}
\mathcal{U}_{1}=\{U \in U(\mathbf{H}): \overline{U(\mathbf{H}) \cdot U}=U(\mathbf{H})\}, \\
\mathcal{U}_{2}=\{U \in U(\mathbf{H}): \text { the spectrum of } U \text { is equal to } \mathbf{T}\}, \\
\mathcal{U}_{3}=\{U \in U(\mathbf{H}): U \text { is multiplicity-free }\}
\end{gathered}
$$

and define $\Sigma_{1}, \Sigma_{2}, \Sigma_{3}$ similarly for $S_{1}(\mathbf{H})$ (also replacing $\mathbf{T}$ by $[-1,1]$ ). By Choksi and Nadkarni $[\mathbf{1}, \mathbf{2}]$ and Simon $[3]$, they are all conjugacy invariant dense $G_{\delta}$ 's.

\section{PROPOSITION 6.7.}

(i) Viewing $\mathbf{H}$ as being $L^{2}\left([0,1), m_{1}\right)$, the mapping $P^{*}(\mathbf{T}) \cap P_{c}(\mathbf{T}) \ni \kappa \mapsto U_{\kappa} \in \mathcal{U}_{2} \cap \mathcal{U}_{3}$ is Borel and $\kappa \sim \kappa^{\prime} \Longleftrightarrow U_{\kappa} \approx U_{\kappa^{\prime}}$, whenever $\kappa, \kappa^{\prime} \in P^{*}(\mathbf{T}) \cap P_{c}(\mathbf{T})$.

(ii) Viewing $\mathbf{H}$ as being $L^{2}\left([0,1], m_{1}\right)$, the mapping $P^{*}([-1,1]) \cap P_{c}([-1,1]) \ni$ $\lambda \mapsto S_{\lambda} \in \Sigma_{2} \cap \Sigma_{3}$ is Borel and $\lambda \sim \lambda^{\prime} \Longleftrightarrow S_{\lambda} \approx S_{\lambda^{\prime}}$, whenever $\lambda, \lambda^{\prime} \in P^{*}([-1,1]) \cap P_{c}([-1,1])$. 
Proof. This follows immediately from Lemma 6.6 and the spectral theorem.

PROpOSITION 6.8.

(i) For any $S \in \Sigma_{2}, U(\mathbf{H}) \cdot S$ is dense in $S_{1}(\mathbf{H})$.

(ii) (Choksi and Nadkarni [1]) For any $U \in \mathcal{U}_{2}, U(\mathbf{H}) \cdot U$ is dense in $U(\mathbf{H})$.

Proof. (i) We will first prove the following claim: let $x_{1}, \ldots, x_{n}$ be $n$ unit vectors in $\mathbf{H}$ such that $1 \leq i<j \leq n \Rightarrow\left|\left(x_{i}, x_{j}\right)\right|<\delta$, where $0<\delta<7^{-n}$. Then the unit vectors $e_{1}, \ldots, e_{n}$ obtained from $x_{1}, \ldots, x_{n}$ by the standard Gram-Schmidt orthogonalization process satisfy the conditions $\left\|x_{i}-e_{i}\right\|<7^{i} \delta, 1 \leq i \leq n$.

By definition, $e_{m}=y_{m} /\left\|y_{m}\right\|$ and $y_{m}=x_{m}-\sum_{k=1}^{m-1}\left(x_{m}, e_{k}\right) e_{k}$ for every $1 \leq m \leq n$, and we argue by induction on $n$. So let $n>1$ and assume the claim for the natural number $n-1$. Then $\left\|x_{i}-e_{i}\right\|<7^{i} \delta, 1 \leq i<n$, and what we need to show is that $\left\|x_{n}-e_{n}\right\|<7^{n} \delta$. But setting $p(n)=\left(\sum_{k=1}^{n-1}\left(1+7^{k}\right)^{2}\right)^{1 / 2}$, we have that $3 p(n)<7^{n}$ and $\left\|y_{n}-x_{n}\right\|^{2}<\delta^{2} p(n)^{2}$, which implies that $1-\delta p(n)<\left\|y_{n}\right\|<1+\delta p(n)$ and

$$
\begin{aligned}
\left\|x_{n}-e_{n}\right\| & =\left\|x_{n}-\frac{x_{n}+y_{n}-x_{n}}{\left\|y_{n}\right\|}\right\| \leq \frac{\left|\left\|y_{n}\right\|-1\right|+\left\|y_{n}-x_{n}\right\|}{\left\|y_{n}\right\|}<\frac{2 \delta p(n)}{1-\delta p(n)} \\
& \leq 3 \delta p(n)<7^{n} \delta .
\end{aligned}
$$

Now let $S \in \Sigma_{2}$ be arbitrary but fixed. If $\left\{t_{n}: n \in \mathbb{N}\right\}$ is any countable dense subset of $[-1,1]$ and $T$ is the unique operator in $S_{1}(\mathbf{H})$ defined by the relations $T \mathbf{e}_{n}=t_{n} \mathbf{e}_{n}(n \in \mathbb{N})$, then, given $N \in \mathbb{N}$, we need only prove that there exists $V \in U(\mathbf{H})$ such that

$$
V S V^{-1} \in\left\{S^{\prime} \in S_{1}(\mathbf{H}):(\forall n \leq N)\left(\left\|\left(S^{\prime}-T\right) \mathbf{e}_{n}\right\|<2^{-N}\right)\right\} .
$$

Since $\sigma(S)=[-1,1]$, the fact that the spectrum of a normal bounded linear operator on $\mathbf{H}$ coincides with its approximate point spectrum implies that there exist unit vectors $\mathbf{x}_{0}, \ldots, \mathbf{x}_{N}$ in $\mathbf{H}$ such that for any $0 \leq n \leq N$,

$$
\left\|S \mathbf{x}_{n}-t_{n} \mathbf{x}_{n}\right\|<\frac{\min \left\{\left|t_{i}-t_{j}\right|: 1 \leq i<j \leq n\right\}}{2^{N+3} \cdot 7^{N+1}} .
$$

Hence, given $0 \leq i<j \leq N$,

$$
\left(t_{i}-t_{j}\right)\left(\mathbf{x}_{i}, \mathbf{x}_{j}\right)=\left(t_{i} \mathbf{x}_{i}-S \mathbf{x}_{i}, \mathbf{x}_{j}\right)+\left(\mathbf{x}_{i}, S^{*} \mathbf{x}_{j}\right)-\left(\mathbf{x}_{i}, t_{j} \mathbf{x}_{j}-S \mathbf{x}_{j}\right)-\left(\mathbf{x}_{i}, S \mathbf{x}_{j}\right),
$$

so

$$
\left|\left(\mathbf{x}_{i}, \mathbf{x}_{j}\right)\right| \leq \frac{\left\|t_{i} \mathbf{x}_{i}-S \mathbf{x}_{i}\right\|+\left\|t_{j} \mathbf{x}_{j}-S \mathbf{x}_{j}\right\|}{\left|t_{i}-t_{j}\right|}<2^{-(N+2)} \cdot 7^{-(N+1)} .
$$

Thus, an application of the claim proved above shows that the unit vectors $\mathbf{u}_{0}, \ldots, \mathbf{u}_{N}$, obtained from $\mathbf{x}_{0}, \ldots, \mathbf{x}_{N}$ by the standard orthogonalization process, satisfy the conditions $\left\|\mathbf{x}_{i}-\mathbf{u}_{i}\right\|<2^{-(N+2)}, 0 \leq i \leq N$. Therefore, by extending $\left\{\mathbf{u}_{0}, \ldots, \mathbf{u}_{N}\right\}$ to a complete orthonormal system $\left\{\mathbf{u}_{n}: n \in \mathbb{N}\right\}$ in $\mathbf{H}$ and setting $V$ to be the unique element of $U(\mathbf{H})$ defined by the relations $V \mathbf{u}_{n}=\mathbf{e}_{n}(n \in \mathbb{N})$, it follows that for any $0 \leq n \leq N$,

$$
\begin{aligned}
\left\|\left(V S V^{-1}-T\right) \mathbf{e}_{n}\right\|= & \left\|S V^{-1} \mathbf{e}_{n}-t_{n} V^{-1} \mathbf{e}_{n}\right\|=\left\|S \mathbf{u}_{n}-t_{n} \mathbf{u}_{n}\right\| \\
= & \left\|S\left(\mathbf{u}_{n}-\mathbf{x}_{n}\right)+\left(S \mathbf{x}_{n}-t_{n} \mathbf{x}_{n}\right)+t_{n}\left(\mathbf{x}_{n}-\mathbf{u}_{n}\right)\right\| \\
\leq & 2\left\|\mathbf{u}_{n}-\mathbf{x}_{n}\right\|+\left\|S \mathbf{x}_{n}-t_{n} \mathbf{x}_{n}\right\|<2 \cdot 2^{-(N+2)} \\
& +2 \cdot 2^{-(N+3)} \cdot 7^{-(N+1)}<2^{-N} .
\end{aligned}
$$




\section{PROPOSITION 6.9.}

(i) (Choksi and Nadkarni [2]) For any $\kappa \in P([-1,1]),\left\{S \in S_{1}(\mathbf{H}): \mu_{S} \perp \kappa\right\}$ constitutes a conjugacy invariant dense $G_{\delta}$ in $S_{1}(\mathbf{H})$, which implies that for any $S \in S_{1}(\mathbf{H}), U(\mathbf{H}) \cdot S$ is meager in $S_{1}(\mathbf{H})$.

(ii) (Choksi and Nadkarni [1]) For any $\lambda \in P(\mathbf{T}),\left\{U \in U(\mathbf{H}): \mu_{U} \perp \lambda\right\}$ constitutes a conjugacy invariant dense $G_{\delta}$ in $U(\mathbf{H})$, which implies that for any $U \in U(\mathbf{H})$, $U(\mathbf{H}) \cdot U$ is meager in $U(\mathbf{H})$.

We are finally in a position to prove Theorem 6.1.

Since $\mathcal{U}_{2} \cap \mathcal{U}_{3}$ constitutes a conjugacy invariant dense $G_{\delta}$ in $U(\mathbf{H})$ and $\Sigma_{2} \cap \Sigma_{3}$ constitutes a conjugacy invariant dense $G_{\delta}$ in $S_{1}(\mathbf{H})$, while the mappings $\Phi: \mathcal{U}_{2} \cap \mathcal{U}_{3} \ni$ $U \mapsto \mu_{U} \in P^{*}(\mathbf{T})$ and $\Phi_{1}: \Sigma_{2} \cap \Sigma_{3} \ni S \mapsto \mu_{S} \in P^{*}([-1,1])$ are continuous and the mappings $\Psi: P^{*}(\mathbf{T}) \cap P_{c}(\mathbf{T}) \ni \kappa \mapsto U_{\kappa} \in \mathcal{U}_{2} \cap \mathcal{U}_{3}$ and $\Psi_{1}: P^{*}([-1,1]) \cap P_{c}([-1,1]) \ni$ $\lambda \mapsto S_{\lambda} \in \Sigma_{2} \cap \Sigma_{3}$ are Borel, Lemma 6.6 and the proof of Theorem 5.1 show that if $Y$ is any Borel $S_{\infty}$-space and $f: U(\mathbf{H}) \rightarrow Y, g: S_{1}(\mathbf{H}) \rightarrow Y$ are any $C$-measurable functions with the property that $U \approx V \Rightarrow f(U) E_{S_{\infty}}^{Y} f(V)$, whenever $U, V$ are in $U(\mathbf{H})$, and $S \approx T \Rightarrow g(S) E_{S_{\infty}}^{Y} g(T)$, whenever $S, T$ are in $S_{1}(\mathbf{H})$, there exist $\sim$-invariant dense $G_{\delta}$ subsets $B$ of $P^{*}(\mathbf{T}) \cap P_{c}(\mathbf{T})$ and $B_{1}$ of $P^{*}([-1,1]) \cap P_{c}([-1,1])$ for which $f[B]$ is contained in a single $E_{S_{\infty}}^{Y}$-class and $g\left[B_{1}\right]$ is contained in a single $E_{S_{\infty}}^{Y}$-class. Thus, setting $A=\Phi^{-1}[B]$ and $A_{1}=\Phi_{1}^{-1}\left[B_{1}\right]$, we obtain unitary conjugacy invariant $G_{\delta}$ subsets of $\mathcal{U}_{2} \cap \mathcal{U}_{3}$ and $\Sigma_{2} \cap \Sigma_{3}$ respectively such that $\Psi[B] \subseteq A$ and $\Psi_{1}\left[B_{1}\right] \subseteq A_{1}$, while the facts that $\mathcal{U}_{2} \subseteq \mathcal{U}_{1}$ and $\Sigma_{2} \subseteq \Sigma_{1}$ (see Proposition 6.8) show that $A$ and $A_{1}$ are also dense in $U(\mathbf{H})$ and $S_{1}(\mathbf{H})$ respectively. Indeed, the implications $U \approx V \in A \Rightarrow \mu_{U} \sim \mu_{V} \in B \Rightarrow$ $\mu_{U} \in B \Rightarrow U \in A$ and $S \approx T \in A_{1} \Rightarrow \mu_{S} \sim \mu_{T} \in B_{1} \Rightarrow \mu_{S} \in B_{1} \Rightarrow S \in A_{1}$ show that $A$ and $A_{1}$ are unitary conjugacy invariant, while the facts that $\Phi \circ \Psi=\mathrm{id}$ and $\Phi_{1} \circ \Psi_{1}=\mathrm{id}$ show that $\Psi[B] \subseteq A$ and $\Psi_{1}\left[B_{1}\right] \subseteq A_{1}$. Therefore, by virtue of part (iv) of the theorem of Hjorth mentioned in the introduction, we need only prove that $A$ and $A_{1}$ are contained in the saturation of $\Psi[B]$ and $\Psi_{1}\left[B_{1}\right]$ respectively according to unitary conjugacy. Indeed, if $U \in A \subseteq \mathcal{U}_{2} \cap \mathcal{U}_{3}$ and $S \in A_{1} \subseteq \Sigma_{2} \cap \Sigma_{3}$, then $\mu_{U}=\Phi(U) \in B \subseteq P^{*}(\mathbf{T}) \cap P_{c}(\mathbf{T})$ and $\mu_{S}=\Phi_{1}(S) \in B_{1} \subseteq P^{*}([-1,1]) \cap P_{c}([-1,1])$; hence $\mu_{\Psi\left(\mu_{U}\right)}=\mu_{U}$ and $\mu_{\Psi_{1}\left(\mu_{S}\right)}=\mu_{S}$ and the spectral theorem implies that $U \approx \Psi\left(\mu_{U}\right) \in \Psi[B]$ and $S \approx \Psi_{1}\left(\mu_{S}\right) \in \Psi_{1}\left[B_{1}\right]$.

Acknowledgements. We would like to acknowledge the support of NSF through grant DMS 9619880. We would also like to thank Tom Wolff for suggesting the argument in the proof of Proposition 5.6(i)(a).

\section{REFERENCES}

[1] J. R. Choksi and M. G. Nadkarni. Baire category in spaces of measures, unitary operators and transformations. Invariant Subspaces and Allied Topics. Eds. H. Helson and S. Yadov. Narosa Publ. Co., New Delhi, 1990, pp. 147-163.

[2] J. R. Choksi and M. G. Nadkarni. Genericity of certain classes of unitary and self-adjoint operators. Canad. Math. Bull. 41(2) (1998), 137-139. 
[3] B. Simon. Operators with singular continuous spectrum: I. General operators. Ann. Math. 141 (1995), 131-145.

[4] A. S. Kechris. Classical Descriptive Set Theory. Springer, 1995.

[5] G. Hjorth, A. S. Kechris and A. Louveau. Borel equivalence relations induced by actions of the symmetric group. Ann. Pure Appl. Logic 92 (1998), 63-112.

[6] G. Hjorth. Classification and Orbit Equivalence Relations (Mathematics Surveys and Monographs, 75). American Mathematical Society, 2000.

[7] A. S. Kechris. Actions of Polish groups and classification problems. Preprint.

[8] D. Preiss and J. Rataj. Maximal sets of orthogonal measures are not analytic. Proc. Amer. Math. Soc. 93(3) (1985), 471-476.

[9] S. Solecki. Polish group topologies. Sets and Proofs (London Mathematics Society Lecture Note Series, 258). Eds. S. B. Cooper and J. K. Truss. Cambridge University Press, 1999, pp. 339-364.

[10] G. Hjorth. Non-smooth infinite dimensional group representations. Preprint.

[11] E. Hewitt and C. Stromberg. Real and Abstract Analysis. Springer, 1965.

[12] R. D. Mauldin, D. Preiss and H. Von Weizsacker. A survey of problems and results concerning orthogonal transition kernels. Measure Theory (Proc. Conf. Oberwolfach, 1981) (Lecture Notes in Mathematics, 945). Springer, 1982.

[13] W. Rudin. Real and Complex Analysis, 3rd edn. WCB/McGraw-Hill, 1987. 